\title{
Specialized Transduction of a Leucine Marker by Proteus mirabilis Phage 5006M
}

\author{
By KATHRYN KRIZSANOVICH-WILLIAMS \\ Microbial Genetics Unit, South African Medical Research Council, \\ Department of Microbiology, University of Pretoria, South Africa
}

(Received 5 June 1975)

\section{INTRODUCTION}

Faulty excision of a prophage from the bacterial chromosome may result in the formation of specialized transducing particles. These carry a bacterial marker which is adjacent to the prophage, but as a rule forfeit part of their genome and this may render them defective (Campbell, 1969). Phages able to form such specialized transducing particles may (e.g. P22 (Zinder \& Lederberg, I952) and PI (Lennox, I955)) or may not (e.g. $\lambda$ (Morse, Lederberg $\&$ Lederberg, 1956 $a$ ) and $\phi 80$ (Matsushiro, 1963)) be capable of general transduction.

Phage $5006 \mathrm{M}$ is a general transducing phage and has given rise to variants which are capable of high frequency transduction of the kanamycin and ampicillin resistance markers of an R factor (Coetzee, 1974, 1975). The host strain Proteus mirabilis PM5006 is cryptically lysogenic for phage 5006M (Krizsanovich, 1973). This cryptic prophage, which is noninducible, is spontaneously liberated on rare occasions and forms turbid plaques on lawns of its parent strain. When strain PM5006 is re-lysogenized by phage 5006M the prophage is inducible. Such strains are lysogenically converted in that they do not readsorb phage $5006 \mathrm{M}$. In this respect host strain PM5006 resembles $P$. mirabilis strain 13 which, on lysogenization with an antigenically identical phage, phage 34 , is also converted to non-adsorption of homologous phage (Coetzee, 196I). It is thought that the re-lysogenizing phage 5006M integrates in tandem to the cryptic prophage (Krizsanovich, 1973). The location of the cryptic prophage is not known. The possibility that inaccurate prophage excision after induction of the double lysogen may give rise to a class of transducing particles which preferentially transduces a locus adjacent to the prophage was investigated.

\section{METHODS}

Organisms. Proteus mirabilis PM5006 (Krizsanovich, 1973; Coetzee, 1974) and various auxotrophs of this strain obtained by treatment with $N$-methyl- $N^{\prime}$-nitro- $N$-nitrosoguanidine (unpublished) were used. The origin of phage 5006 M has been described (Krizsanovich, I973).

Media and methods. Media, prophage induction by u.v. light, lytic phage preparation and general phage methods were according to Krizsanovich (1973). Transductions to prototrophy and segregation studies were done by the methods of Coetzee (I974). Phage 5006M antiserum with a neutralization constant of $\mathrm{I} 80 \mathrm{~min}^{-1}$ was used in some transduction experiments and in segregation studies to prevent reinfection. 
Table I. Transduction frequencies of markers by phage $5006 \mathrm{M}$ lysates prepared by induction and infection

$\begin{array}{lcc}\text { Marker } & \text { Induced lysate } & \begin{array}{c}\text { Lysate from } \\ \text { infection }\end{array} \\ \text { PM5006ura } & 2 \times 10^{-7} & 1 \times 10^{-7} \\ \text { PM5006trp } & 2 \times 10^{-7} & 3 \times 10^{-7} \\ \text { PM5006orn } & 5 \times 10^{-8} & 1 \times 10^{-8} \\ \text { PM5006his } & 2 \times 10^{-7} & 2 \times 10^{-7} \\ \text { PM5006leu-I } & 3 \times 10^{-4} & 1 \times 10^{-6} \\ \text { PM5006leu-2 } & 1 \times 10^{-4} & 5 \times 10^{-7} \\ \text { PM5006leu-3 } & 2 \times 10^{-4} & 7 \times 10^{-7}\end{array}$

One $\mathrm{ml}$ of an exponentially-growing culture was infected with phage at a m.o.i. of I. After adsorption for $\mathrm{I} 5 \mathrm{~min}$ at $37^{\circ} \mathrm{C}$, the mixtures were washed once in $0.85 \%(\mathrm{w} / \mathrm{v}) \mathrm{NaCl}$ and appropriate dilutions plated on minimal medium.

\section{RESULTS}

A lysate of PM5006 obtained by infection, and an induced lysate of the double lysogen PM5006(5006M) were used to transduce a series of auxotrophs to prototrophy. Frequencies of transduction for a number of markers are given in Table I. No abortive transductants were encountered and transductant clones were not surrounded by a halo of growth indicative of transduction occurring on the plates (Smith-Keary, 1966). The transduction frequencies of the induced lysate equalled those of the lysate obtained by infection for all markers except the three independently-isolated leucine-requiring strains which were transduced about 300 times more efficiently by the induced lysate. Seven further lysates induced from independently-prepared double lysogens of PM5006 also transduced leucine independence at frequencies of $\mathrm{I} \times \mathrm{IO}^{-4}$ to $4 \times \mathrm{IO}^{-4}$ /plaque-forming unit (p.f.u.) adsorbed, similar to the initial induced lysate. Ten transductants formed at a multiplicity of infection (m.o.i.) of 0.0004 were purified by streaking for single colonies. These transductants, which arose from a single infection and were protected from reinfection by the use of phage antiserum after adsorption, were lysogenic and liberated wild-type phage spontaneously and after u.v. induction. None were able to plate phage $5006 \mathrm{M}$, due to lack of adsorption as a result of lysogenic conversion by the transducing phage (Krizsanovich, 1973; Coetzee, 1974). This indicates that the phage carrying the leucine marker did not lose the ability to convert its host. Segregation studies on these lysogenic transductants yielded an average of I \% leucine auxotrophs after overnight growth in broth containing phage-specific antiserum. All transductants and auxotrophic segregants were stably lysogenic. The frequency of transduction was independent of m.o.i. up to an infection of about I. Multiplicities of infection greater than 2 were accompanied by a doubling of transduction frequency (Fig. I). The fall in transduction frequency between m.o.i. of 2 and 0.4 is too steep to be indicative of mutual aid between transducing particles (Chan et al. 1972). Homologous non-transducing helper phage at a m.o.i. of 2 had a similar effect (Fig. I). The formation of transductants is therefore helper-independent. Lysates prepared by u.v. induction of transductants also transduced the leucine marker at the same frequency as the original induced lysates. 


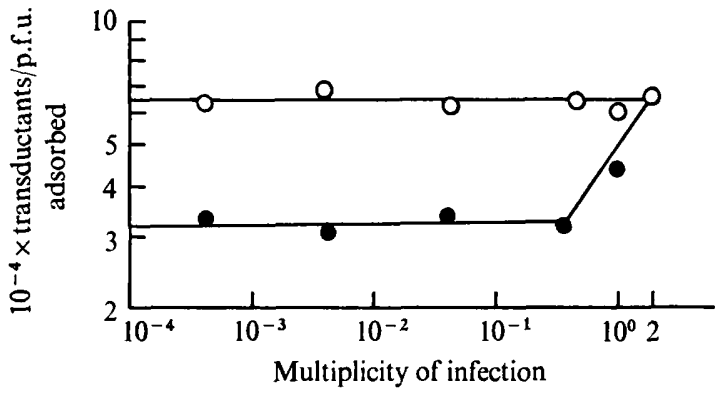

Fig. I. Effect of helper phage on transduction frequency. Dilutions of the induced lysate were made in a constant concentration of a lysate of PM5006leu-I produced by lytic infection. The m.o.i. of the non-leucine transducing phage was 2 p.f.u. $\bullet$, Induced lysate alone; $\bigcirc$, with helper phage.

\section{DISCUSSION}

Transduction of the leucine marker by phage 5006M resembles high frequency transduction of kanamycin and ampicillin resistance by variants of this phage (Coetzee, 1974, 1975) in that transductants which arose from single infection were lysogenic, converted to phage non-adsorption and their formation was helper-independent. Due to the high segregation rate, these transductants were almost certainly heterogenotes (Morse, Lederberg \& Lederberg, $1956 b$ ) formed by a single cross-over with the bacterial chromosome. The leucine transduction frequency was lower than the corresponding antibiotic resistance transduction frequencies and non-transducing phage exerted a less pronounced helper effect. The presence of the cryptic prophage in strain PM5006 makes it difficult to ascertain the extent to which the leucine transducing phage is defective (see Coetzee, 1974). Since specialized transducing particles were only present in induced lysates they were probably formed by faulty excision of the prophage on induction. In its additional capacity for general transduction (Table I) the induced lysate resembles the phages PICM (Kondo \& Mitsuhashi, 1964) and P22-p-pro (Jessop, 1972). Phage P22-p-pro differs, however, in that the specialized transducing particles were formed during lytic infection. Most high frequency transducing (h.f.t.) lysates are produced in two stages (see Morse et al. 1956a; Matsushiro, 1963; SmithKeary, 1966): an initial lysate transduces with low frequency, and induction of the resulting transductants gives rise to h.f.t. lysates. Specialized transduction of leucine by phage $5006 \mathrm{M}$ differs in that lysates induced from wild-type double lysogens and from transductants transduced with similar frequencies.

The formation of specialized transducing phages by induction of lysogens represents a method for locating a prophage on the chromosome. Since all lysates induced from independently-prepared double lysogens of PM5006 showed specialized transduction of only the leucine marker, it is suggested that phage $5006 \mathrm{M}$ integrates near the leu locus.

\section{REFERENCES}

Campbell, A. (1969). Episomes. New York: Harper \& Row.

Chan, R. K., Botstein, D., Watanabe, T. \& Ogata, Y. (1972). Specialized transduction of tetracycline resistance by phage $\mathrm{P}_{22}$ in Salmonella typhimurium. Virology 50, 883-898.

CoETzeE, J. N. (196I). Lysogenic conversion in the genus Proteus. Nature, London 189, 946-947.

CoetzeE, J. N. (1974). High frequency transduction of kanamycin resistance in Proteus mirabilis. Journal of General Microbiology 84, 285-296. 
CoetzeE, J. N. (1975). High frequency transduction of resistance to ampicillin and kanamycin in Proteus mirabilis. Journal of General Microbiology 87, 173-176.

JESSOP, A. P. (1972). A specialized transducing phage of $\mathbf{P 2 2}$ for which the ability to form plaques is associated with transduction of the proAB region. Molecular and General Genetics 114, 214-222.

Kondo, E. \& MitsuHASHI, S. (1964). Drug resistance of enteric bacteria. IV. Active transducing bacteriophage PICM produced by the combination of R factor with bacteriophage PI. Journal of Bacteriology 88, $1266-1276$.

Krizsanovich, K. (1973). Cryptic lysogeny in Proteus mirabilis. Journal of General Virology 19, 3I I-320.

LENNOX, E. S. (1955). Transduction of linked genetic characters of the host by bacteriophage PI. Virology $\mathbf{r}$, 190-206.

MatsushiRo, A. (1963). Specialized transduction of tryptophan markers in Escherichia coli $\mathrm{KI} 2$ by bacteriophage $\phi 80$. Virology 19, 475-482.

MorSE, M. L., LeDerberg, E. M. \& LeDerberg, J. (1956a). Transduction in E. coli KI 2. Genetics 4I, I42156.

MoRSE, M. L., LeDerberg, E. M. \& LeDerberg, J. (1956b). Transductional heterogenotes in Escherichia coli. Genetics 4I, 758-779.

Smith-Keary, P. F. (I966). Restricted transduction by bacteriophage P22 in Salmonella typhimurium. Genetical Research 8, 73-82.

ZiNDER, N. D. \& LeDERBERG, J. (1952). Genetic exchange in Salmonella. Journal of Bacteriology 64, 679699. 